\title{
Implementing canny edge detection algorithm for noisy image
}

\author{
Ehsan Akbari Sekehravani ${ }^{1}$, Eduard Babulak ${ }^{2}$, Mehdi Masoodi ${ }^{3}$ \\ ${ }_{1,3}$ Young Researchers and Elite Club, Iran \\ ${ }^{2}$ Department of Computer Science, Liberty University, USA
}

\begin{tabular}{l}
\hline \hline Article Info \\
\hline Article history: \\
Received Oct 15, 2019 \\
Revised Feb 3, 2020 \\
Accepted Mar 15, 2020 \\
\hline
\end{tabular}

Keywords:

Canny algorithm

Edge detection

Image processing

Median filter

Noisy image

\begin{abstract}
Edge detection is a significant stage in different image processing operations like pattern recognition, feature extraction, and computer vision. Although the Canny edge detection algorithm which exhibits high precision is computationally more complex contrasted to other edge detection methods. Due to the traditional Canny algorithm uses the Gaussian filter, which gives the edge detail represents blurry also its effect in filtering salt-and-pepper noise is not good. To resolve this problem, we utilized the median filter to maintain the details of the image and eliminate the noise. This paper presents implementing and also enhancing the accuracy of Canny edge detection for noisy images. Results present that this proposed method can definitely overcome noise disorders, preserve the edge useful data, and likewise enhance the edge detection precision.
\end{abstract}

This is an open access article under the CC BY-SA license.

\section{Corresponding Author:}

Ehsan Akbari Sekehravani,

Young Researchers and Elite Club, Iran.

Email: akbarisekkehravani@gmail.com

\section{INTRODUCTION}

Image processing has been developed basically for two purposes. The first is for better interpretation of images by humans and the second is for analysis, data storage, and transfer. Significant improvements have been made to image processing. Image processing is used in many areas such as security, medical imaging and surveillance, retrieval and more. There are many uses in image processing such as object detection, feature extraction, image analysis, where they have image segmentation as a pre-processing [1, 2]. Edge detection is a widely used operation in image processing and plays a very important role in identifying and separating geometric shapes in the image. The edges always contain important information from the image. The objects in the image are separated by the edges of the image. Thus it can be said that one of the most significant steps in machine vision is edge detection [3].

Fingerprint identification [4], car-license plate [5], fire alarm systems [6], image processing based SAR [7] are some usual fields in which edge detection (ED) is used. Canny [8], and Prewitt and Sobel are the most commonly used algorithm in the ED. Dictionary learning $[9,10]$ and fractional derivatives $[11,12]$ are other areas in these ED plays the main role. Implementing ED in Morphological operators [13, 14] and detectors transformation $[15,16]$ leads to acceptable consequences. One of the most common uses in image analysis is edge detection because the edge is the boundary between an object and its background, that is, the edge of the two gray levels or two-pixel brightness values that occur at a particular location of the image. As this level changes more, edge recognition will be easier.

This paper aims to implement and enhance the accuracy of the Canny edge detection algorithm for noisy images thus we used the median filter for decreasing the noise in edges once they are detected by the Canny algorithm. It can be mentioned that in future projects this algorithm will be implemented in localization problems [17] to improve the accuracy. The paper is organized as follows. The edge detection is 
presented in section 2. Section 3 is about the Canny edge detection algorithm. We present implementing the Canny edge detection algorithm using the median filter in 4, experimental results in section 5 and conclusion in section 6 .

\section{EDGE DETECTION}

Dots in the image that have sudden brightness changes are called edges. Edge points usually include object boundaries and other types of brightness changes, as well as noise edges. There are various edges such as inclined, stairs, ceiling and rising edges. Because the edges of the objects in the image contain extremely beneficial information, therefore, edge detection algorithms are the main research method [18]. Edge detection is a type of image segmentation technique that identifies edges in an image. The edges are also represented as a collection of interconnected points located at the border between the two areas. The edges illustrate the borders of the object, and hence this feature can be used to segment an image toward its primary areas or objects.

The image is a compound of different intensities of brightness, noise and more. The edge in an image is the overall change in brightness intensity. The image is divided into different areas or sections by edges. Edges are the border among segments or regions. The purpose of detecting edges is to extract image information that contains useful information, for instance, shape, position, the object size in the image, image sharpness, and enrichment. There are several edge detection algorithms in image processing such as Canny, Sobel, Prewitt, Robert. Among these algorithms, the performance of the Canny edge detection algorithm is better than others. The Canny edge detection algorithm will present in the next section.

\section{CANNY EDGE DETECTION ALGORITHM}

To improve the edge detection process the Canny edge detection algorithm was introduced. For this goal, three fundamental criteria were considered. The detection of all the principal edges in the reference image was the first and significant criterion. The main purpose was to reduce the error in the edges. The localization was the second criterion that it is detected edge points as close as possible to the correct edge. In the third criterion, it was not to possess rather than one response to a single edge. The third step was performed because the first two cases were not important enough to eliminate the probability of rather than one response to an edge.

Since the Canny algorithm can keep a low error rate, preserving useful information by filtering out spam and likewise maintaining fewer changes from the main image and eliminating multiplex responses to the near edge, as a result, this algorithm is one of the best known. Based on this criterion, the Canny edge detection algorithm operates as follows [19]. The Canny algorithm runs in five separate steps.

\subsection{Noise detection by smoothing}

The outcomes of edge detection are extremely sensitive to image noise because the mathematics involved behind the scenes is essentially based on derivatives. One method to obtain rid of the noise on the image is by implementing a Gaussian blur to smooth it. To accomplish this, the image concentration method with a Gaussian kernel $(3 \times 3,5 \times 5,7 \times 7$, etc. $)$ is used. The size of the core depends on the trace of the expected blur. Fundamentally, the less visible is the blur for the smallest kernel. In this step, first, the input image needs to be converted into a grayscale by adjusting contrast and brightness so that the image is blurred to eliminate noise. Therefore, for making the location and detection edge effective, the first step is to filter for removing noise in the main image. Commonly, it is used as a Gaussian filter for noise elimination [20]. In 2-D, the common equation of Gaussian filter can be defined as follows:

$$
G(x \cdot y)=\frac{1}{2 \pi \sigma^{2}} \exp \left(-\frac{x^{2}+y^{2}}{2 \sigma^{2}}\right)
$$

where $\mathrm{y}$ is the distance from the origin in the vertical axis, $\mathrm{x}$ is the distance from the origin in the horizontal axis and $\sigma$ is the standard deviation of the Gaussian distribution.

\subsection{Calculate image gradient}

The gradient computation step by calculating the image gradient using the Canny edge detection operator detects the edge and direction intensities. By calculating the image gradient, the edge pixels with drastic changes in gray area values are identified. Since points in the direction of most intensity variation represent so the gradient is a unit vector. At this stage, first, the vertical and horizontal ingredients of 
the gradient are calculated and then it is computed the magnitude and orientation of the gradient [1]. The gradient magnitude $G$ and gradient angle $\Theta$ are calculated as follows:

$$
\begin{aligned}
& \text { Gradient Magnitude }=G=\sqrt{\left(G_{x}^{2}+G_{y}^{2}\right)} \\
& \text { Gradient Angle }=\theta=\text { are } \tan \left(\frac{G_{y}}{G_{x}}\right)
\end{aligned}
$$

$G_{x}$ and $G_{y}$ represent the horizontal and vertical gradients respectively.

Canny edge detection algorithm utilizes four filters to calculate diagonal, vertical and horizontal edges in the blurred image. Also, the edge direction angle is rounded to one of the four angles demonstrating vertical, horizontal and the two diagonals $\left(0^{\circ}, 45^{\circ}, 90^{\circ}, 135^{\circ}\right.$ degrees $)$. It results to estimate the first derivative in the vertical direction $G_{y}$ and horizontal $G_{x}$ [21]. Then, the edges where the intensity of the gray level changes the most are detected by the Canny algorithm.

\subsection{Non-maxima suppression (NMS)}

This method is based on one of the two techniques generally employed for edge detection, the first one is to consider edges as the zero-crossings of the Laplacian of image intensity [22, 23]. The second one is to suppress the local non-maxima of the magnitude of the gradient of image intensity in the direction of this gradient [23] also this method called NMS. Ideally, the ultimate image should possess slim edges. Thus, to thin the edges, we need to fulfill NMS. Also, NMS can effectively find the edge and repress the occurrence of incorrect edges.

NMS is also based on the gradient magnitudes that the detector converts the thick edges of the image, to nearly thin and sharp edges which can be more utilized for identification purposes. It is mainly performed in NMS for thinning the edge. In this process, the image is scanned along the edge direction and rejects any pixel value that is not considered to be an edge which will result in a thin line in the output image.

\subsection{Double thresholding}

The threshold value based on 2 parts, T1=high threshold, T2=low threshold. If the pixels possessing values of a grayscale level higher than $\mathrm{T} 1$ are strong edge pixels, the result is edge area. The result is the nonedge region if the pixels having values of a grayscale level less than T2 are weak edge pixels. The result is depending on the neighboring pixels If the pixels possess values of the grayscale level among T1 and T2 [24]. The stage goal at identifying three types of pixels: strong, weak, and non-relevant. In this paper, we used this step as follows:

- Pixels which they have an intensity value and not enough to be considered, are weak pixel, but they are small enough to be considered as irrelevant edge detection.

- Pixels with high intensity are strong ones in which they participate in the final edge.

- Other pixels contribute as non-relevant for the edge.

Also, we can see what the double thresholds maintain for:

- The low threshold is applied to detect irrelated pixels (intensity less than the low threshold).

- Pixels which they have an intensity value and not enough to be considered, are weak pixel, but they are small enough to be considered as irrelevant edge detection.

- The high threshold is utilized to recognize the strong pixels (intensity higher than the high threshold).

- All pixels possessing intensity among both thresholds are flagged as weak and the Hysteresis system (next stage) will aid us to identify the ones that could be regarded as strong and the ones that are regarded as irrelevant.

\subsection{Track edge by hysteresis}

Edges will remove in the output image if they do not link to a very definitive edge. If weak edges that are connected with strong edges, they will contain in the ultimate image. Strong edges are represented as certain edges and are contained in the final edge image. A pixel is labeled as a strong edge pixel if its gradient magnitude is bigger than the upper threshold. Whereas if the value of the gradient magnitude of the pixel falls among the lower and higher threshold, a pixel is classified as a weak edge pixel. Strong edges are the ones which can be included immediately as edges in the final edge image. On the other hand, if weak edges are linked to the strong edges, they can be marked. 


\section{IMPLEMENTING CANNY EDGE DETECTION USING MEDIAN FILTER}

Due to the traditional Canny algorithm uses Gaussian filtering, which makes the edge detail seems blurry also its effect in filtering salt-and-pepper noise is not good. To solve this problem, the median filter can be used to preserve the details of the image and remove the noise. Image Noise is classified as Amplifier noise (Gaussian noise), Salt-and-pepper noise(Impulse noise), Shot noise, Quantization noise (uniform noise), on-isotropic noise, Speckle noise (Multiplicative noise) and Periodic noise. The edges found using the traditional Canny edge algorithm may have noise present in it. Usually, the presentment of noise may give a wrong edge. Therefore, in this paper, we try to increase the reliability of edge detection by noise elimination. There are several non-linear ways to overcome impulse noise like maximum(max), minimum(min) and median filters [25]. Amongst all of these filters, the median filter is beneficial for overcoming noise, such as salt and pepper noise which may create a challenge in edge detection.

To eliminate noise frequently median filter, which is a non-linear filter, is utilized. Noise decline is a common preprocessing stage to enhance the outcomes of the subsequent processing, for example, edge detection on an image, signal processing and smoothers for image processing. Since under specific conditions, this filter maintains edges whilst eliminating noise thus it is popularly used in digital image processing. The fundamental idea of this filter is to operate through the signal entry through the entry, changing any entry with the median of neighboring entries. The median filter can remove the effect of input noise magnitudes with very large values that this trait is a significant benefit of the median filter over linear filters. To implement the first idea, different images are damaged by a known noise such as salt and pepper noise. In this paper, the median filter algorithm is used to replace the Gaussian filtering. In this method, the improvement of edges with a compound of the median filter should produce much better results which are presented in the next section. After applying the median filter, the proposed method follows the usual steps: image smoothing and filtering, finding the gradient magnitude and gradient direction, non-maxima suppression and edge tracking by hysteresis. The pixel value of a given point in an image is replaced by an average value in its neighborhood, which not only reduces noise penetration however can likewise eliminate the isolated point [22].

\section{EXPERIMENTAL RESULTS}

Although the digital image has a low-frequency spectrum, both the edge and the noise are high-frequency signals. As a result, the noise in the image causes the edge detection algorithm to mistake the edge detection and produce poor results. In this section, we have presented the results achieved by using the traditional Canny algorithm and our proposed method on various images. The proposed method for edge detection in noisy images was implemented in MATLAB R2015a. Experiments were performed on the platform Intel(R) Core(TM) i5-4210U CPU at 1.70GHz, 4GB RAM, Microsoft Windows 10 Enterprise. First, original images, which is Figure 1, are damaged by a distinguished noise such as salt and pepper noise, that noisy images are illustrated in Figure 2, for implementing the proposed method. Figure 2 shows that the noisy images where the noise is available in terms of white and black points. These black and white points are known as salt and pepper noise.

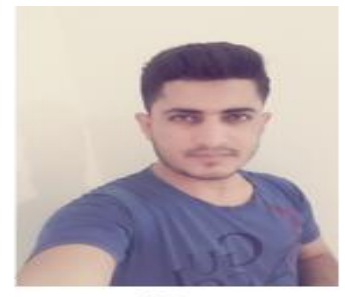

(a)

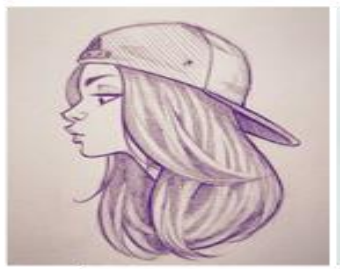

(c)

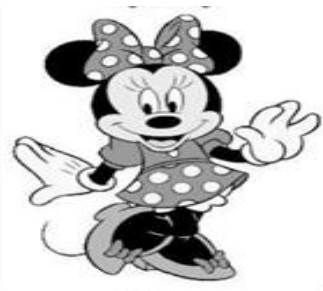

(b)

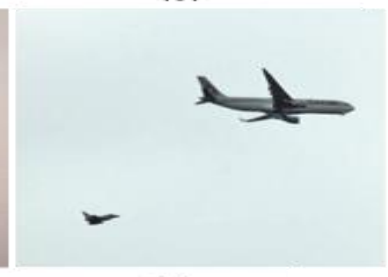

(d)

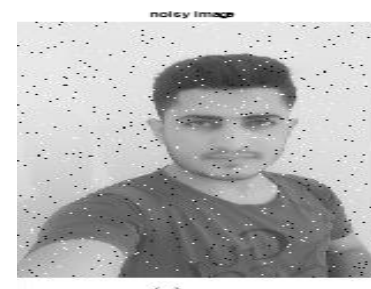

(a)

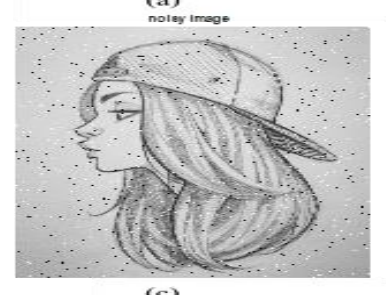

(c)

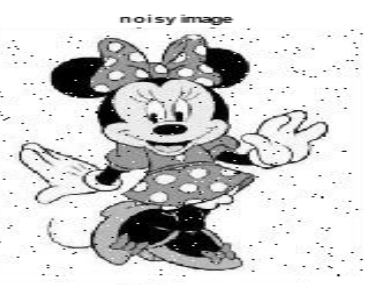

(b)

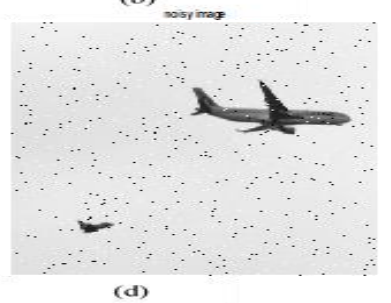

Figure 2. Noisy images, (a) Man, (b) Carton, (c) Drawing, (d) Aircraft

Figure 1. Original images, (a) Man, (b) Carton, (c) Drawing, (d) Aircraft 
Then the edges of the noisy images are detected before utilizing the median filter using the traditional Canny edge detector as shown in Figure 3. Obviously, the noise will disturb the detected edges, to improve this condition median filter is applied upon noisy images. To examine our suggested method, four different images were applied.
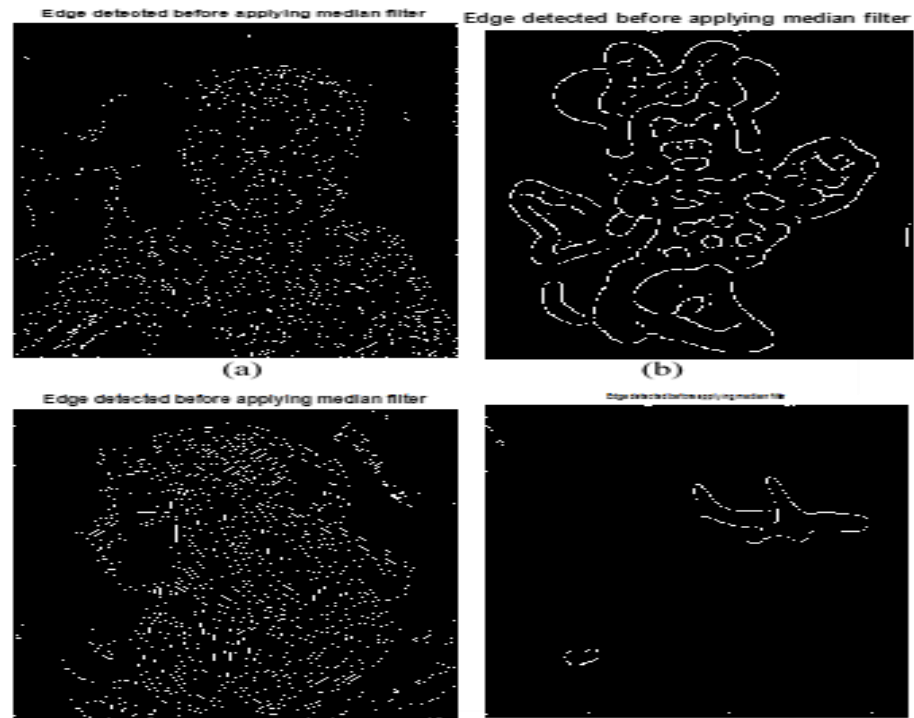

(c)

(d)

Figure 3. Edge detected using Canny algorithm before implementing median filter

Next, the median filter implemented on the noisy images as illustrated in Figure 4. Hence, the median filter accomplishes on noisy images, it removes the noise thus the images are visually better than the noisy images and the results of edge recognition also are improved. Also, Figure 5. presents the edge detected in the images after using the median filter. Consequently, according to the results, our proposed method worked better.

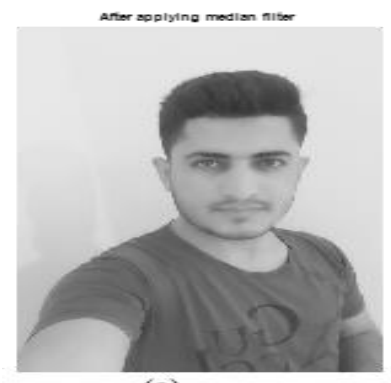

(a)

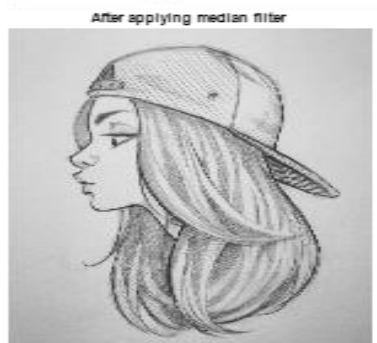

(c)

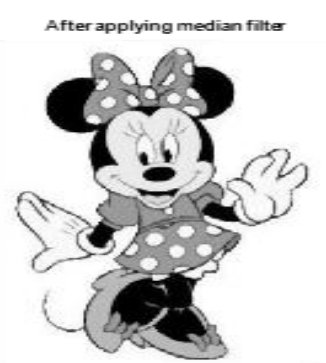

(b)

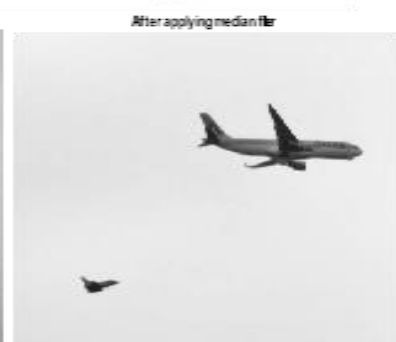

(d)

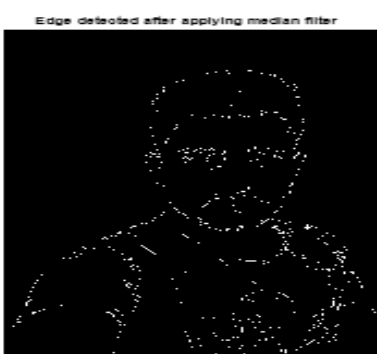

(a)

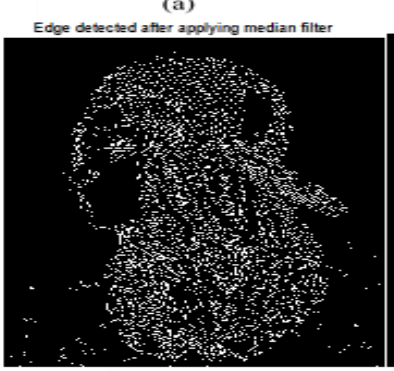

(c)
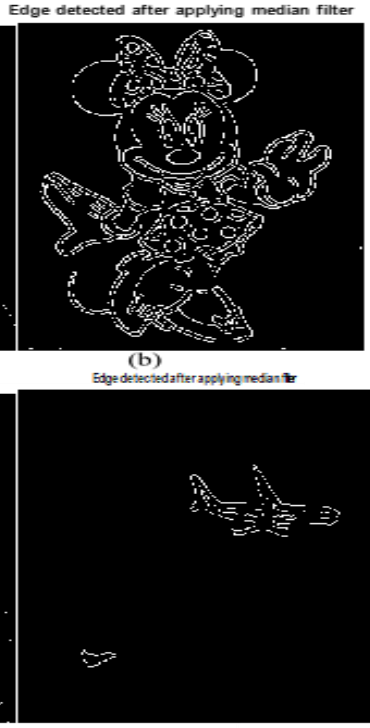

(d)

Figure 4. Implementing median filter

Figure 5. Edge detected using the Canny algorithm after implementing the median filter 


\section{CONCLUSION}

In conclusion, there is usually noise in all the edge detected and the edges detected are not continuous before implementing the median filter. Thus the noise-reducing method is essential because traditional Canny edge detection is very sensitive to noise. Canny edge detection algorithm gives the most excellent outcomes in a noisy environment whereas its implementation is complex and has a high expenditure. Experimental results have shown that with the proposed method, edges in noisy images can be detected successfully and better in edge and detail detection than standard Canny algorithm. Likewise, enhance the edge detection precision.

\section{REFERENCES}

[1] Rezai-Rad, "Comparison of SUSAN and Sobel Edge Detection in MRI Images for Feature Extraction," in Information and Communication Technologies, ICTTA, vol. 6, pp. 1103-1107, 2006.

[2] Geng Xin, C. Ke and H. Xiaoguang, "An improved Canny edge detection algorithm for color image," in Industrial Informatics (INDIN), pp. 113-117, July 2012.

[3] X. H. a. W. Jia, "Hexagonal structure for intelligent vision," in in First International Conference on Information and Communication Technologies, ICICT, Aug. 2005.

[4] C. H. Y. D. C. L. J. Chen, "Combining fractional-order edge detection and chaos synchronisation classifier for fingerprint identification," IET Image Processing, vol. 8, pp. 354-362, 2014.

[5] S. M. A. R. R. A. I. A. M. Al-Ghaili, "Vertical-edgebased car-license-plate detection method," IEEE Transactions on Vehicular Technology, vol. 62, pp. 26-38, 2013.

[6] T. Y. G. L. T. Qiu, "An autoadaptive edge-detection algorithm for flame and fire image processing," IEEE Transactions on Instrumentation, vol. 61, pp. 1486-1493, 2012.

[7] Y. X. J. Y. C. Liu, "A coastline detection method in polarimetric SAR images mixing the region-based and edge-based active contour models," IEEE Transactions on Geoscience and Remote Sensing, vol. 55, pp. 3735-3747, 2017.

[8] J. Canny, "A computational approach to edge detection," IEEE Transactions on Pattern Analysis and Machine Intelligence, vol. 8, pp. 679-698, 1986.

[9] J. Mairal, M. Leordeanu, F, Bach, M. Hebert, J. Ponce "Discriminative sparse image models for class-specific edge detection and image interpretation," in Proceedings of European Conference on Computer Vision, Marseille, France, pp. 43-56, 2008.

[10] G. Z. Q. Wang, "Ore image edge detection using HOG-index dictionary learning approach," The Journal of Engineering, pp. 542-543, 2017.

[11] S. A. M. H. K. Vignesh, "Edge detection using Fractional derivatives and Information sets," Journal of Electronic Imaging, vol. 27, 2018.

[12] E. A. A. Nandal, "Image edge detection using fractional calculus with feature and contrast enhancemen," Circuits, Systems, and Signal Processing, vol. 37, pp. 3946-3972, 2018.

[13] M. K. Y.P. B. Chanda, “A multi-scale morphologic edge detector,” Pattern Recognition, vol. 31, pp. 1469-1478, 1998.

[14] F. W. P. Shui, "Anti-impulse-noise edge detection via anisotropic morphological directional derivatives," IEEE Transactions on Image Processing, vol. 26, pp. 4962-4977, 2017.

[15] W. H. S. Mallat, "Singularity detection and processing with wavelets," IEEE Transactions on Information Theory, vol. 32, pp. 617-643, 1992.

[16] G. A. M. K. M. M.R. Hassanzadeh, "Two new methods for finding endocardial and epicardial boundaries in echocardiographic images using wavelet analysis," European Journal of Scientific Research, vol. 27, pp. 264-274, 2009.

[17] Mehdi Masoodi, Ehsan Akbari Sekehravani, Mohsen Maesoumi, "Rssi-Based Modified K-Nearest Neighbors Algorithm For Indoor Target Tracking," Far East Journal of Electronics and Communications, vol. 18, pp. 345-356, 2018.

[18] O. P. V, "An optimal fuzzy system for edge detection in color images using bacterial foraging algorithm," IEEE Transactions on Fuzzy Systems, pp. 114-127, 2017.

[19] A. L. K. a. D. V. Sangam, "Canny edge detection algorithm," International Journal of Advanced Research in Electronics and Communication Engineering (IJARECE), vol. 5, pp. 1292-1295, 2016.

[20] R. M. a. D. Aggarwal, "Study and Comparison of Various Image Edge Detection Techniques," International Journal of Image Processing (IJIP), vol. 3, 2009.

[21] J.-M. J. a. F. C. C. Wolf, "Text localization, enhancement and binarization in multimedia documents," in Object recognition supported by user interaction for service robots, Quebec City, Quebec, Canada, vol. 2, pp. 1037-1040, 2002.

[22] R. Haralick, "Digital step edges from zero crossing of second directional," IEEE Transactions on Pattern Analysis and Machine Intelligence, pp. 58-68, January 1984.

[23] R. M. Haralick and L. G. Shapiro, Computer and Robot Vision, Addison-Wesley, 1992.

[24] T. J. a. T. C. T. Kitti, "An Object Recognition and Identification System Using the Harris Corner Detection Method," International Journal of Machine Learning and Computing, vol. 2, pp. 462-465, August 2012.

[25] Rafael C. Gonzalez,Richard E. Woods, 3rd ed, "Digital Image Processing, Pearson Education Publication," 2009. 


\section{BIOGRAPHIES OF AUTHORS}
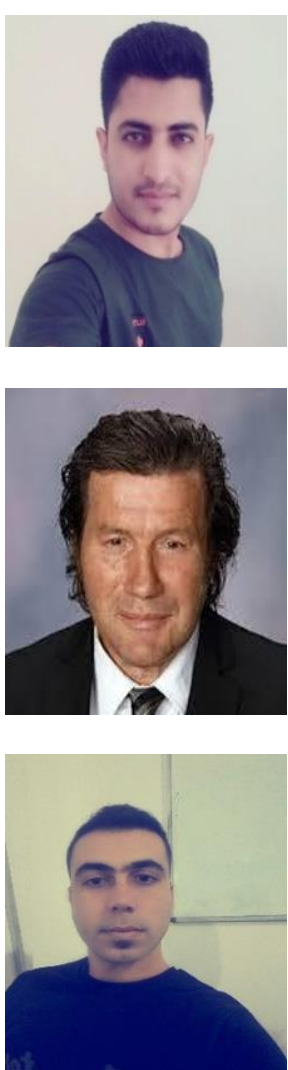

Ehsan Akbari Sekehravani was born in Iran in 1990. He received the M.S.c degree in Telecommunications Engineering from Islamic Azad University, Jahrom Branch, Iran, in 2016. $\mathrm{He}$ is currently a Ph.D. student in Electromagnetic engineering at the University of Campania Luigi Vanvitelli, Aversa, Italy. His main fields of interest are Image processing, Signal processing, Inverse Scattering problems, and Inverse source problems.

Professor Eduard Babulak received the D.Sc. degree from the University of Pardubice (Czech Republic), 2008 and also the Ph.D. degree from the Staffordshire University (United Kingdom), 2003. He is currently a Professor of Computer Science and Cyber Security, Liberty University, USA. He is an accomplished international scholar, researcher, consultant, educator, professional engineer and polyglot, with more than thirty years of experience.

Mehdi Masoodi was born in Iran in 1987. He received the M.S.c degree in Telecommunications Engineering from Islamic Azad University, Kazerun Branch, Iran, in 2015. He is currently a Ph.D. student in Electromagnetic engineering at the University of Campania Luigi Vanvitelli, Aversa, Italy. His main fields of interest are Signal processing, Image processing, Inverse Scattering problems, and Inverse source problems. 